\title{
ÜBER DISKONTINUIERLICHE GRUPPEN PICARD- SCHEN TYPUS UND ZUGEHÖRIGE EISENSTEINSCHE REIHEN
}

\author{
TOMIO KUBOTA
}

Herrn Professor Kiyoshi Noshiro zu seinem 60. Geburtstag gewidmet

Die vorliegende Arbeit ist eine Zusammenfassung einiger Tatsachen und Formeln betreffs einer Transformationsgruppe, die diskontinuierlich auf dem dreidimensionalen euklidischen Halbraum operiert. Eine solche Gruppe, die zum erstenmal in [5] betrachtet wurde, heiße hier eine diskontinuierliche Gruppe Picardschen Typus. Die in dieser Arbeit angegebenen Resultate ergeben sich grundsätzlich aus der allgemeinen Theorie der algebraischen Gruppen sowie der symmetrischen Räume, und aus der entsprechenden Theorie der Eisensteinschen Reihen. ([1], [2], [4], [6], [7]). Deswegen stellen wir im folgenden die einzelnen Behauptungen meistens nur ohne Beweise auf. Für die Anwendung auf die zukünftigen Arbeiten des Verfassers, [3] z. B., ist es trotzdem zweckmäßig, einen Teil der Theorie der diskontinuierlichen Gruppen Picardschen Typus in einer konkreten, spezialisierten Form zusammenzufassen, wie bei der diskontinuierlichen Gruppe Fuchsschen Typus. Denn es gibt wenige Artikel über die Picardsche Gruppe und folglich ist es oft schwierig, selbst die einfachen Formeln, wie die in dieser Arbeit stehenden, aus der Literatur zu entnehmen.

\section{§ 1. Der obere Halbraum.}

Man betrachte zuerst die Gruppe $G=S L(2, C)$ aller komplexen $2 \times 2$ Matrizen mit Determinante 1. Die Gruppe $U=S U(2)$ aller unitären $2 \times 2$ Matrizen mit Determinante 1 ist dann eine maximale kompakte Untergruppe von $G$, und der homogene Raum $H=G / U$ ist ein über $\boldsymbol{R}$ dreidimensionaler, symmetrischer Raum. Für ein beliebiges Element $\omega=\left(\begin{array}{ll}x_{11} & x_{12} \\ x_{21} & x_{22}\end{array}\right) \in G$, gibt es eine eindeutige Zerlegung von der Form

Received June 17, 1967. 


$$
\omega=\left(\begin{array}{ll}
1 & z \\
& 1
\end{array}\right)\left(\begin{array}{ll}
\sqrt{v} & \\
& \frac{1}{\sqrt{v}}
\end{array}\right) \omega_{0}
$$

mit $z \in \boldsymbol{C}, \quad 0<v \in \boldsymbol{R}$, und $\omega_{0} \in U$ (Iwasawa Zerlegung), so daß man $u=(z, v)$ als eine Koordinatendarstellung des natürlichen Bildes $u=u(\omega) \in H$ eines Elementes $\omega \in G$ auffassen kann. Es ist aber bequemer, die Matrizenbezeichnung $u=\left(\begin{array}{rr}z & -v \\ v & \bar{z}\end{array}\right)$ statt $u=(z, v)$ zu benutzen, weil dann die Operation $u \rightarrow \sigma u$ von $\sigma=\left(\begin{array}{ll}a & b \\ c & d\end{array}\right) \in G$ auf $H$ in der Form

$$
\sigma u=(\tilde{a} u+\tilde{b})(\tilde{c} u+\tilde{d})^{-1}
$$

der gewöhnlichen linearen Transformation geschrieben werden kann, wobei die Schlange die Abbildung $\boldsymbol{C} \ni x \rightarrow \tilde{x}=\left(\begin{array}{ll}x & \\ & \bar{x}\end{array}\right)$ bedeutet. Es sei ferner $j=\left(\begin{array}{ll}1 & -1 \\ 1 & \end{array}\right)$ d dann ist

$$
u(\omega)=\omega j
$$

Andererseits wird $u(\omega)=\left(\begin{array}{rr}z & -v \\ v & \bar{z}\end{array}\right)$ für $\omega=\left(\begin{array}{ll}x_{11} & x_{12} \\ x_{21} & x_{22}\end{array}\right)$ explizite durch

$$
z=\frac{x_{11} \overline{x_{21}}+x_{12} \overline{x_{22}}}{x_{21} \overline{x_{21}}+x_{22} \overline{x_{22}}}, \quad v=\frac{1}{x_{21} \overline{x_{21}}+x_{22} \overline{x_{22}}}
$$

angegeben, und es gilt noch

(3) $\quad \sigma u=\left(\begin{array}{cc}(a z+b)(\bar{c} \bar{z}+\bar{d})+a \bar{c} v^{2}, & -v \\ v & (\bar{a} \bar{z}+\bar{b})(c z+d)+\bar{a} c v^{2}\end{array}\right)\left(|c z+d|^{2}+|c|^{2} v^{2}\right)^{-1}$

für $u=\left(\begin{array}{rr}z & -v \\ v & \bar{z}\end{array}\right) \in H, \quad \sigma=\left(\begin{array}{ll}a & b \\ c & d\end{array}\right) \in G$.

Dem Raum $H$, der jetzt schon als der dreidimensionale, obere Halbraum realisiert ist, geben wir eine Struktur als ein nichteuklidischer, hyperbolischer Raum mit der $G$-invarianten Riemannschen Metrik

$$
\frac{1}{v^{2}}\left(d x^{2}+d y^{2}+d v^{2}\right)
$$

Dann ist

$$
d \mu(u)=\frac{d x d y d v}{v^{3}}
$$


das $G$-invariante Maß, und der Operator von Laplace-Beltrami in bezug auf die Riemannsche Metrik ist

$$
D=v^{2}\left(\frac{\partial^{2}}{\partial x^{2}}+\frac{\partial^{2}}{\partial y^{2}}+\frac{\partial^{2}}{\partial v^{2}}\right)-v \frac{\partial}{\partial v} ;
$$

er ist der wesentlich einzige $G$-invariante Differentialoperator von $H=G / U$.

Die Operation (1) kann natürlich auf jede Matrix $\left(\begin{array}{rr}z & -v \\ v & \bar{z}\end{array}\right)$ mit beliebigem $v \in \boldsymbol{R}$ angewandt werden. In der Weise operiert $G$ auch auf dem ganzen Raum $\boldsymbol{R}^{3}$, und dabei wird $\boldsymbol{R}^{3}$ in drei $G$-transitive Bestandteile getrennt. Die drei Bestandteile sind nämlich der obere Halbraum, der untere Halbraum, und die $z$-Ebene mit $v=0$. Wir fügen der $z$-Ebene, die offensichtlich mit $\boldsymbol{C}$ identifiziert werden kann, noch den unendlich fernen Punkt $\infty$ hinzu; sie bildet eine komplexe projektive Linie. Wegen dieses Verfahrens erhält man in der Tat eine glatte Form der Operation von $G$ auf $\boldsymbol{R}^{3}$.

Im folgenden betrachten wir nicht die Gruppe $G$ selbst, sondern hauptsächlich die Gruppe der Transformationen, welche die Elemente von $G$ auf $\boldsymbol{R}^{3}$ verwirklichen. In der Hinsicht ist es sinnvoll, zu verabreden, daß ein Element $\sigma \in G$ stets als eine verkürzte Schreibweise des von $\sigma$ vertretenen Elementes aus $G /\left\{ \pm\left(\begin{array}{ll}1 & \\ & 1\end{array}\right)\right\}=P S L(2, \boldsymbol{C})$ betrachtet werden muß.

Wir erwähnen jetzt die Klassifizierung der Elemente von $G$. Einem Element $\sigma \in G$ wird der Gestalt der Jordanschen Normalform von $\sigma$ entsprechend einer der folgenden Namen gegeben:

\begin{tabular}{ccc} 
& \multicolumn{1}{c}{ Name } & Normalform \\
I) & Einselement & $\left(\begin{array}{ll}1 & \\
& 1\end{array}\right)$ \\
II) & elliptisches Element & $\left(\begin{array}{ll}\varepsilon & \\
& \bar{\varepsilon}\end{array}\right)$ \\
III) & hyperbolisches Element & $\left(\begin{array}{cc}v & \\
& v^{-1}\end{array}\right)$ \\
IV) & loxodromisches Element & $\left(\begin{array}{cc}v \varepsilon & \\
& v^{-1} \bar{\varepsilon}\end{array}\right)$ \\
V) & parabolisches Element & $\left(\begin{array}{cc}1 & z \\
& 1\end{array}\right)$
\end{tabular}

Hierbei sind $\boldsymbol{C} \ni \varepsilon \neq \pm 1, \quad|\varepsilon|=1, \quad \boldsymbol{R} \ni v>0, \quad v \neq 1$, und $\boldsymbol{C} \ni z \neq 0 . \quad$ Die 
geodätischen Linien in unserem Raum $H$ sind die Kreisbogen oder Geraden, die auf der z-Ebene senkrecht stehen. Ein elliptisches, hyperbolisches, oder loxodromisches Element aus $G$ bildet genau eine geodätische Linie von $H$ auf sich ab. Während dabei ein elliptisches Element eine geodätische Linie punktweise fest läßt, haben dagegen die beiden anderen nur zwei Punkte aus $C \cup\{\infty\}$ als Fixpunkte. Deshalb ist $\sigma \in G$ elliptisch, wenn nur $\sigma$ einen Fixpunkt in $H$ hat. Ein parabolisches Element hat nur einen Punkt von $\boldsymbol{C} \cup\{\infty\}$ als Fixpunkt.

\section{§2. Diskontinuierliche Gruppen Picardschen Typus.}

Eine diskrete Untergruppe $\Gamma$ von $G=S L(2, \boldsymbol{C})$ heißt eine diskontinuierliche Gruppe Picardschen Typus, oder kurz eine diskontinuierliche Gruppe. Die Gruppe $\Gamma$ muß dabei, wie in $\$ 1$ verabredet wurde, als eine Gruppe von Transformationen von $H=G / U$ betrachtet werden. Zwei Punkte $u_{1}, u_{2} \in \boldsymbol{R}^{3}$ werden äquivalent in bezug auf $\Gamma$ genannt, wenn $u_{1}=r u_{2}$ für ein $\gamma \in \Gamma$ besteht.

Enthält eine diskontinuierliche Gruppe $\Gamma$ ein elliptisches Element $\sigma \in G$, so muß $\sigma$ von endlicher Ordnung sein. Ist andererseits $c \in \boldsymbol{C} \cup\{\infty\}$ der Fixpunkt eines parabolischen Elementes aus $G$, so gibt es kein hyperbolisches oder loxodromisches Element von $G$, das $c$ als Fixpunkt hat. Diese beiden Eigenschaften sind sehr elementar zu schließen. Der gemeinsame Fixpunkt zweier unabhängiger parabolischen Elemente in $\Gamma$ heißt eine Spitze von $\Gamma$.

Ist $u_{0} \in H$, und ist $\Gamma$ eine diskontinuierliche Gruppe, so ist die Menge $\Gamma u_{0}$ aller $r u_{0}$ mit $r \in \Gamma$ eine diskrete Menge von $H$. Daraus erkennt man, daß es einen Fundamentalbereich $\mathscr{D}=\Gamma \backslash H=\Gamma \backslash G / U$ von $\Gamma$ gibt, der eine Teilmenge von $H$ ist. Wenn man ferner voraussetzt, daß die Menge der in $\boldsymbol{C} \cup\{\infty\}$ liegenden Grenzpunkte eines Fundamentalbereichs $\mathscr{D}$ von $\Gamma$ nur aus Spitzen von $\Gamma$ besteht, so kann man zeigen, daß man den Fundamentalbereich von $\Gamma$ als Polyeder mit nur endlich vielen Seiten im nichteuklidischen Raum $H$ wählen kann. Einen solchen Fundamentalbereich erhält man am einfachsten als die Menge aller Punkte $u \in H$, für welche die Entfernung zwischen $u$ und $u_{0}$ in bezug auf die Metrik (4) kleiner ist als die Entfernung zwischen $u$ und $\gamma u_{0}$ für alle $\gamma \in \Gamma$ mit $\gamma \neq 1$. Hierbei muß aber $u_{0}$ die Bedingung erfüllen, daß $\gamma u_{0}=u_{0}$ nur für $\gamma=1$ gilt. Mittels eines so konstruierten Fundamentalbereichs erhält man ohne große Schwierigkeit mehrere wichtige Ergebnisse, $u$. a., die endliche Erzeugbarkeit 
von $\Gamma$, und die Eindlichkeit des invarianten Maßes $A(\mathscr{D})$ in bezug auf (5) des Fundamentalbereichs $\mathscr{D}=\Gamma \backslash H$.

Eine diskontinuierliche Gruppe $\Gamma$, für welche die Grenzpunkte eines Fundamentalbereichs nur Spitzen sind, und die folglich die oben angegebenen Endlichkeitseigenschaften hat, nennen wir eine diskontinuierliche Gruppe endlichen Typus.

Ein wichtiges Beispiel einer diskontinuierlichen Gruppe endlichen Typus ist die Gruppe $\Gamma=S L(2, \mathfrak{v})$ aller $2 \times 2$ Matrizen mit Determinante 1, deren Komponenten zum Ring $\mathfrak{v}$ der ganzen Zahlen des Gaußschen Zahlkörpers $\boldsymbol{Q}(\sqrt{-1})$ gehören. Ein Fundamentalbereich $\mathscr{D}$ von $\Gamma$ ist durch die folgenden Bedingungen bestimmt [5]:

$$
0 \leqq x+y, \quad x \leqq \frac{1}{2}, \quad y \leqq \frac{1}{2}, \quad 1 \leqq x^{2}+y^{2}+v^{2}
$$

Der einzige Grenzpunkt von $\mathscr{D}$ ist die Spitze $\infty$.

Es sei $\Gamma$ eine diskontinuierliche Gruppe endlichen Typus, $c$ eine Spitze von $\Gamma$, und es sei $\Gamma_{c}$ die Gruppe aller $\gamma \in \Gamma$ mit $\gamma_{c}=c$. Die sämtlichen parabolischen Elemente in $\Gamma_{\mathbf{c}}$ bilden dann eine zu $\boldsymbol{Z} \times \boldsymbol{Z}$ isomorphe Gruppe $\Gamma_{0}$, und der Index $\left(\Gamma_{c}: \Gamma_{0}\right)$ ist endlich. Es sei nun $\chi$ ein Charakter von $\Gamma, d . h$. eine Abbildung von $\Gamma$ in $C$ mit $\chi(\sigma \tau)=\chi(\sigma) \chi(\tau),|\chi(\sigma)|=1$, $(\sigma, \tau \in \Gamma)$. Dann heißt $c$ eine wesentliche Spitze von $\Gamma$ in bezug auf $\chi$, wenn $\chi\left(\Gamma_{\mathrm{c}}\right)=1$ ist.

\section{§3. Eisensteinsche Reihen.}

Es sei $\Gamma$ eine diskontinuierliche Gruppe endlichen Typus, für die $\infty$ eine Spitze ist. Man nehme an, daß die Gruppe $\Gamma_{\infty}$ aller $\gamma \in \Gamma$ mit $\gamma \infty=\infty$ zu $\boldsymbol{Z} \times \boldsymbol{Z}$ isomorph ist, ${ }^{1)}$ und daß der euklidische Flächeninhalt von $\Gamma_{\infty} \backslash \boldsymbol{C}$ $=P$ gleich 1 ist. Hier ist $P$ sozusagen ein Periodenparallelogramm von $\Gamma_{\infty}$. Es sei ferner $\chi$ ein Charakter von $\Gamma$ derart, daß $\infty$ eine wesentliche Spitze von $\Gamma$ in bezug auf $\chi$ ist. Für eine komplexe Veränderliche $s$ definiert man dann die Eisensteinsche Reihe $E(u, s, \chi)$ durch

$$
E(u, s, \chi)=\sum_{\sigma \in \Gamma_{\infty} \backslash \Gamma} \bar{\chi}(\sigma) v(\sigma u)^{s}
$$

1) Diese Beschränkung ist nicht wesentlich sondern dient nur der Einfachheit der Beschreibung. 
wobei $v(u)=v$ für $u=\left(\begin{array}{rr}z & -v \\ v & \bar{z}\end{array}\right) \in H$ ist. Die Eisensteinsche Reihe ist dann und nur dann absolut konvergent, wenn $\operatorname{Re} s>2$ ist, und ist eine holomorphe Funktion von $s$ für $\operatorname{Re} s>2$. Die folgenden beiden Eigenschaften sind eine unmittelbare Folge der Definition:

$$
\begin{aligned}
& E(\sigma u, s, \chi)=\chi(\sigma) E(z, s, \chi), \quad(\sigma \in \Gamma), \\
& D E(z, s, \chi)=\lambda E(z, s, \chi)
\end{aligned}
$$

mit

$$
\lambda=s(s-2) .
$$

Nach der Voraussetzung enthält $\Gamma_{\infty}$ kein elliptisches Element, und folglich hat jedes Element $\sigma \in \Gamma_{\infty}$ die Form $\sigma= \pm\left(\begin{array}{ll}1 & b \\ & 1\end{array}\right) . \quad$ Sind andererseits $\sigma=\left(\begin{array}{ll}a & b \\ c & d\end{array}\right), \quad \sigma^{\prime}=\left(\begin{array}{ll}a^{\prime} & b^{\prime} \\ c & d\end{array}\right)$ zwei Elemente aus $\Gamma$, so sieht man ohne weiteres $\sigma \sigma^{\prime-1}=\left(\begin{array}{c}1, a^{\prime} b-a b^{\prime} \\ 1\end{array}\right) \in \Gamma_{\infty}$. Daraus ergibt sich, daß für zwei Elemente $\sigma=\left(\begin{array}{ll}a & b \\ c & d\end{array}\right), \quad \sigma^{\prime}=\left(\begin{array}{ll}a^{\prime} & b^{\prime} \\ c^{\prime} & d^{\prime}\end{array}\right)$ aus $\Gamma$ die Gleichung $\Gamma_{\infty} \sigma=\Gamma_{\infty} \sigma^{\prime}$ dann und nur dann gilt, wenn für die Zeilenvektoren $(c, d)= \pm\left(c^{\prime}, d^{\prime}\right)$ gilt. Also gibt es eine eineindeutige Zuordnung zwischen den Restklassen in $\Gamma_{\infty} \mid \Gamma$ und den Zahlenpaaren $(c, d) \operatorname{mit}\left(\begin{array}{ll}* & * \\ c, & d\end{array}\right) \in \Gamma$ modulo \pm 1 . Da der Wert $\chi(\sigma)=\chi(c, d)$ von $\chi$ bei $\sigma=\left(\begin{array}{ll}a & b \\ c & d\end{array}\right)$ auch nur durch $(c, d)$ bestimmt wird, hat man jetzt nach (3), (8) eine konkrete Formel

$$
E(u, s, \chi)=\frac{1}{2} \sum_{\substack{c, d \\
\left(\begin{array}{ll}
* & * \\
c & d
\end{array}\right) \in \Gamma}} \bar{\chi}(c, d) \frac{v^{s}}{\left(|c z+d|^{2}+|c|^{2} v^{2}\right)^{s}}
$$

für die Eisensteinsche Reihe.

Die Summation ist über alle diejenigen Paare $(c, d)$ komplexer Zahlen zu erstrecken, für die mindestens ein Element von der Form $\pm\left(\begin{array}{ll}* & * \\ c & d\end{array}\right)$ in $\Gamma$ existiert.

Man betrachte nun den dualen Modul $\Gamma_{\infty}^{*}$ von $\Gamma_{\infty}, d . h$. die additive Gruppe aller $m \in \boldsymbol{C}$ mit $\operatorname{tr} \tilde{m} \tilde{b}=m b+\bar{m} \tilde{b} \in \boldsymbol{Z}$ für alle $b \in \Gamma_{\infty}$, wobei ein Element $\left(\begin{array}{ll}1 & b \\ & 1\end{array}\right) \in \Gamma_{\infty} \quad$ durch die Zuordnung $b \leftrightarrow\left(\begin{array}{ll}1 & b \\ & 1\end{array}\right)$ mit der komplexen Zahl $b$ identifiziert werden muß. Dann ist $\Gamma_{\infty}^{*}$ auch ein Gitter 
in $\boldsymbol{C}$, und die Eisensteinsche Reihe hat als eine zweifach periodische Funktion von $z$ die Fourierentwicklung

$$
E(u, s, \chi)=\sum_{m \in \Gamma_{\infty}^{*}} a_{m}(v, s, \chi) e^{2 \pi i \operatorname{tr} \tilde{m} \bar{z}}
$$

mit

$$
a_{m}(v, s, \chi)=\int_{P} E(u, s, \chi) e^{-2 \pi i \operatorname{tr} m z} d x d y
$$

Ist $m \neq 0$, so ist $a_{m}$ eine Art Besselscher Funktion von $v$, was man aus der von (10) stammenden Differentialgleichung

$$
\frac{d^{2} a_{m}}{d v^{2}}-\frac{1}{v} \frac{d a_{m}}{d v}-\left(16 \pi^{2}|m|^{2}+\frac{\lambda}{v^{2}}\right) a_{m}=0
$$

für $a_{m}$ leicht ablesen kann.

Das konstante Glied $a_{0}$ hat eine besondere Wichtigkeit für die Funktionalgleichung Eisensteinscher Reihen. Als eine explizite Formel für $a_{0}$ bekommt man durch eine direkte Berechnung

$$
a_{0}(v, s, \chi)=v^{s}+\phi(s, \chi) v^{2-s}
$$

mit



wobei die Kongruenz $d_{1} \equiv d_{2}(\bmod c)$ für zwei komplexe Zahlen durch $d_{1}-d_{2}=c b$ mit $b \in \Gamma_{\infty}$ definiert wird. Wegen $\left(\begin{array}{ll}* & * \\ c & d\end{array}\right)\left(\begin{array}{ll}1 & b \\ & 1\end{array}\right)=\left(\begin{array}{cc}* & * \\ c & d+b c\end{array}\right)$ hängt $\chi(c, d)$ tatsächlich nur der Restklasse von $d \bmod c$ ab.

Ein Hauptsatz in der Theorie der Eisensteinschen Reihen lautet nun, daß die zunächst für $\operatorname{Re} s>2$ definierte Funktion (8) auf die ganze $s$-Ebene meromorph fortsetzbar ist. Außerdem ist auch dabei bewiesen, daß $E(u, s, \chi)$ sowie $\phi(s, \chi)$ für $\operatorname{Re} s \geqq 1$ holomorph ist bis auf endlich viele Pole erster Ordnung, die dem Intervall $1<s \leqq 2$ angehören.

\section{§4. Funktionalgleichung.}

Wir betrachten jetzt eine diskontinuierliche Gruppe $\Gamma$ endlichen Typus mit einem Charakter $\chi$ von $\Gamma$. Die wesentlichen Spitzen in bezug auf $\chi$ eines Fundamentalbereichs $\mathscr{D}=\Gamma \backslash H$ bezeichnen wir mit $\kappa_{1}, \kappa_{2}, \cdots, \kappa_{h}$. 
Ferner setzen wir voraus, daß für jedes $i$ die Gruppe $\Gamma_{i}$ aller $\gamma \in \Gamma$ mit $\gamma_{\kappa_{i}}=\kappa_{i}$ zu $\boldsymbol{Z} \times \boldsymbol{Z}$ isomorph ist. Es sei nun $\sigma_{i}$ ein Element von $G \mathrm{mit}$ $\sigma_{i} \infty=\kappa_{i}$ und zwar mit der Eigenschaft, daß der Flächeninhalt eines Periodenparallelogramms $P_{i}=\Gamma_{i, \infty} \mid \boldsymbol{C}$ der nach Voraussetzung zu $\boldsymbol{Z} \times \boldsymbol{Z}$ isomorphen Gruppe $\Gamma_{i, \infty}=\sigma_{i}^{-1} \Gamma_{i} \sigma_{i}$ gleich 1 ist. Wir definieren dann durch

$$
E_{i}(u, s, \chi)=\sum_{\sigma \in \Gamma_{i} \mid \Gamma} \bar{\chi}(\sigma) v\left(\sigma_{i}^{-1} \sigma u\right)^{s}, \quad(\operatorname{Re} s>2),
$$

die Eisensteinsche Reihe $E_{i}(u, s, \chi)$ für die wesentliche Spitze $\kappa_{i}$. Für beliebiges $i, j$ ist $E_{i}\left(\sigma_{j} u, s, \chi\right)$ eine zweifach periodische Funktion von $z$, und mittels des dualen Moduls $\Gamma_{j, \infty}^{*}$ von $\Gamma_{j, \infty}$ erhält man also wei in $\S 3$ eine Fourierentwicklung

$$
E_{i}\left(\sigma_{j} u, s, \chi\right)=\sum_{m \in \Gamma_{j, \infty}^{*}} a_{i j, m}(v, s, \chi) e^{2 \pi i \operatorname{tr} \tilde{m} z}
$$

mit

$$
a_{i j, m}(v, s, \chi)=\int_{P_{j}} E_{i}\left(\sigma_{j} u, s, \chi\right) e^{-2 \pi i \operatorname{tr} \tilde{m} z} d x d y \quad \text { 2) }
$$

Diese Fourierentwicklung heißt Fourierentwicklung von $E_{i}(u, s, \chi)$ bei der Spitze $\kappa_{j}$.

Die Transformation $\sigma_{i}$ ist nicht eindeutig bestimmt, sondern an ihrer Stelle kann jede Transformation von der Form $\sigma_{i}^{\prime}=\sigma_{i}\left(\begin{array}{rr}1 & w \\ & 1\end{array}\right)\left(\begin{array}{ll}\varepsilon & \\ & \bar{\varepsilon}\end{array}\right) \operatorname{mit}|\varepsilon|$ $=1, w \in C$, und nur solche, benutzt werden. Dabei ändert $E_{i}(u, s, \chi)$ sich nicht, und in (19), (20) wird $\Gamma_{j, \infty}^{*}$ bezw. $P_{j}$ durch $\varepsilon^{2} \Gamma_{j, \infty}^{*}$ bezw. $\bar{\varepsilon}^{2} P_{j}$ ersetzt. Daraus ergibt sich insbesondere, daß das konstante Glied $a_{i j, 0}(v, s, \chi)$ von der Wahl von $\sigma_{i}$ unabhängig ist.

Die Funktion $E_{i}\left(\sigma_{i} u, s, \chi\right)$ ist bis auf Bezeichnungen mit der in $\S 3$ untersuchten $E(u, s, \chi)$ identisch. Deshalb können alle analytischen Eigenschaften, z. B. die analytische Fortsetzung in bezug auf $s$, von $E_{i}(u, s, \chi)$ schon als erledigt verstanden werden. Die Formeln (9), (10) gelten auch unverändert für $E_{i}(u, s, \chi)$.

Wegen

$$
E_{i}\left(\sigma_{j} u, s, \chi\right)=\sum_{\sigma \in \Gamma_{i}, \infty i \sigma_{i}^{-1} \Gamma \sigma_{j}} \bar{\chi}\left(\sigma_{i} \sigma \sigma_{j}^{-1}\right) v(\sigma z)^{s}
$$

2) Solange dadurch keine Verwechselung verursacht wird, werde der Buchstabe $i$ für die beiden Zwecke, als $\sqrt{-1}$ und als ein Index, verwendet. 
hat man außerdem eine (12) ähnliche Formel

$$
E_{i}\left(\sigma_{j} u, s, \chi\right)=\frac{1}{2} \sum_{\substack{c, d \\
\left(\begin{array}{c}
* \\
c,
\end{array}\right) \in \sigma_{i}^{-1} \Gamma \sigma_{j}}} \bar{\chi}_{i j}(c, d) \frac{v^{s}}{\left(|c z+d|^{2}+|c|^{2} v^{2}\right)^{s}}
$$

mit

$$
\chi_{i j}(c, d)=\chi\left(\sigma_{i}\left(\begin{array}{ll}
a & b \\
c & d
\end{array}\right) \sigma_{j}^{-1}\right)
$$

Betreffs der Bedeutung der Summationszeichen soll die Verabredung in $\S 3$ beachtet werden.

Aus (23) erhält man wie bei (16), (17) eine explizite Angabe des konstanten Gliedes

$$
a_{i j, 0}(v, s, \chi)=\left\{\begin{array}{rr}
v^{s}+\phi_{i i}(s, \chi) v^{2-s}, & i=j, \\
\phi_{i j}(s, \chi) v^{2-s}, & i \neq j,
\end{array}\right.
$$

mit

$$
\phi_{i j}(s, \chi)=\frac{\pi}{s-1} \cdot \frac{1}{2} \sum_{\substack{c \neq 0 \\
\left(\begin{array}{c}
* \\
c, c
\end{array}\right) \in \sigma_{i}^{-1} \Gamma \sigma_{j}}} \frac{1}{|c|^{2 s}}\left(\sum_{d \bmod c} \bar{\chi}_{i j}(c, d)\right) .
$$

Für die nichtwesentlichen Spitzen sind die zugehörigen konstanten Glieder nicht direkt $\mathrm{zu}$ definieren.

Setzt man jetzt

$$
\Phi(s, \chi)=\left(\phi_{i j}(s, \chi)=\left(\begin{array}{cccc}
\phi_{11} & \phi_{12} & \cdots & \phi_{1 h} \\
\phi_{21} & \phi_{22} & \cdots & \phi_{2 h} \\
\cdots & \cdots & \cdots \\
\phi_{h 1} & \phi_{h 2} & \cdots & \phi_{h h}
\end{array}\right),\right.
$$

und

$$
\mathscr{E}(u, s, \chi)=\left(\begin{array}{c}
E_{1}(u, s, \chi) \\
E_{2}(u, s, \chi) \\
\vdots \\
E_{h}(u, s, \chi)
\end{array}\right)
$$

so ist wieder nach einem Hauptsatz der Theorie der Eisensteinschen Reihen $\Phi(1+i t, \chi), \quad(t \in \boldsymbol{R})$, eine unitäre Matrix, und es gilt 


$$
\Phi(s, \chi) \Phi(2-s, \chi)=I, 3)
$$

sowie die Funktionalgleichung der Eisensteinschen Reihe

$$
\mathscr{E}(u, s, \chi)=\Phi(s, \chi) \mathscr{E}(u, 2-s, \chi)
$$

\section{§5. Weitere Folgerungen der Funktionalgleichung.}

Die Funktionalgleichung der Eisensteinschen Reihe kann natürlich verstanden werden als die Gesamtheit aller Funktionalgleichungen, denen die einzelnen Glieder in der Fourierentwicklung der Eisensteinschen Reihe genüge leisten. Die Funktionalgleichung für das kontakte Glied ist dabei nichts anderes als (28). Im folgenden werden wir diejenige Funktionalgleichung etwas genauer untersuchen, welche unmittelbar aus (29) folgt, wenn man ein nichtkonstantes Glied der Fourierentwicklung der Eisensteinschen Reihe betrachtet.

Die Fourierkoeffizienten $a_{i j, m}(v, s, \chi)$ in (20) lassen sich ähnlich wie bei (16) folgenderweise schreiben:

$$
a_{i j, m}(v, s, \chi)=v^{2-s} \phi_{i j}(s, \chi, m) K(2 m v, s)
$$

mit

$$
\phi_{i j}(s, \chi, m)=\frac{1}{2} \sum_{c \neq 0} \frac{1}{|c|^{2 s}}\left(\sum_{\substack{d \bmod c \\
\left(\begin{array}{ll}
* & * \\
c & d
\end{array}\right) \in \sigma_{i}^{-1} \Gamma \sigma_{j}}} \bar{\chi}_{i j}(c, d) e^{\pi i \operatorname{tr} \tilde{m} \tilde{d} / \tilde{c}}\right),
$$

und

$$
K(w, s)=\int_{-\infty}^{\infty} \int_{-\infty}^{\infty} \frac{e^{-\pi i t r w z}}{\left(x^{2}+y^{2}+1\right)^{s}} d x d y, \quad(z=x+i y, w \in C) .
$$

Dieses Integral hat zunächst nur für $\operatorname{Re} s>1$ einen wohlbestimmten Sinn. Durch partielle Integration wird jedoch $K(w, s)$ in die ganze $s$-Ebene fortgesetzt, und zwar ist es eine ganze Funktion, wenn nur $w \neq 0$ ist. Ist $\varepsilon$ eine komplexe Zahl mit $|\varepsilon|=1$, so folgt $K(\varepsilon w, s)=K(w, s)$ aus (32). Also hat man

$$
K(w, s)=K(|w|, s)
$$

Die Funktion $K(u, s)$ genügt in der Tat wieder einer Art von Funkti-

3) Die Einheitsmatrix. 
onalgleichung. Obwohl die Funktionalgleichung direkt, rein analytisch bewiesen werden kann, ist sie von unserem Standpunkt auch folgenderweise erreichbar. Man betrachte nämlich $\Gamma=S L(2, \mathfrak{v})$, wie sie in $\S 2$ erklärt wurde, und man nehme den trivialen Charakter $\chi=1$ von $\Gamma$. Dann ist

$$
E(u, s, \chi)=\frac{1}{4} \sum_{\substack{c, d \in \mathfrak{D} \\(c, d)=1}} \frac{v^{s}}{\left(|c z+d|^{2}+|c|^{2} v^{2}\right)^{s}}
$$

die einzige Eisensteinsche Reihe, ${ }^{4}$ ) und wegen (24) ist

$$
\Phi(s, \chi)=(\phi(s))
$$

mit

$$
\phi(s)=\frac{\pi}{s-1} \frac{\zeta(s-1)}{\zeta(s)}
$$

wobei $\zeta(s)$ die Dedekindsche Zetafunktion des Körpers $\boldsymbol{Q}(\sqrt{-1})$ ist. Da der duale Modul im Sinne des $\S 3$ die Differente von $\boldsymbol{Q}(\sqrt{-1})$ ist, hat $E(u, s, \chi)$ die Fourierentwicklung von der Form

$$
E(u, s, \chi)=\sum_{m \in \mathfrak{v}} a_{m}(v, s, \chi) e^{\pi i t r \tilde{m} \tilde{z}},
$$

und wegen (30) sowie (31) mit $\frac{1}{4}$ statt $\frac{1}{2}$ gilt

$$
\begin{aligned}
& a_{1}(v, s, \chi)=v^{2-s} \cdot \sum_{0 \neq c \in 0} \frac{\mu(c)}{N c^{s}} \cdot K(v, s) \\
= & v^{2-s} \zeta(s)^{-1} K(v, s)
\end{aligned}
$$

wobei $\mu(c)$ die sogenannte Möbiussche Funktion bedeutet. Aus der Funktionaleichung

$$
E(u, s, \chi)=\phi(s) E(u, 2-s, \chi)
$$

folgt jetzt

$$
a_{1}(v, s, \chi)=\phi(s) a_{1}(v, 2-s, \chi)
$$

und es gilt deshalb

4) Der vor dem Summenzeichen stehende Faktor stammt von der Tatsache, daß $\Gamma_{\infty}$ in diesem Fall nicht zu $\boldsymbol{Z} \times \boldsymbol{Z}$ isomorph ist. Dadurch kommt aber nichts wesentlich neues heraus. Alle bisher bewiesenen Formeln gelten auch für diesen Fall, evtl. mit einer kleinen Änderung des konstanten Faktors. 


$$
v^{2-s} \zeta(s)^{-1} K(v, s)=\frac{\pi}{s-1} \frac{\zeta(s-1)}{\zeta(s)} \cdot v^{s} \zeta(2-s)^{-1} K(v, 2-s)
$$

Mittels (33), und mittels der Funktionalgleichung

$$
Z(s)=\pi^{2 s-1} \frac{\Gamma(1-s)}{\Gamma(s)} Z(1-s) \text { 5) }
$$

der Dedekindschen Zetafunktion erhält man also

$$
K(w, s)=\pi^{2 s-2}|w|^{2 s-2} \frac{\Gamma(2-s)}{\Gamma(s)} K(w, 2-s)
$$

für jedes $w \in \boldsymbol{C}, \quad(w \neq 0)$, als Funktionalgleichung von $K$.

Nun kommen wir zum allgemeinen Fall zurück und betrachten die beiden $m$-ten Glieder der Fourierentwicklung auf den beiden Seiten von (29). Man erhält sofort

$$
\left(\begin{array}{c}
a_{1 j, m}(v, s, \chi) \\
a_{2 j, m}(v, s, \chi) \\
\vdots \\
a_{h j, m}(v, s, \chi)
\end{array}\right)=\Phi(s, \chi)\left(\begin{array}{c}
a_{1 j, m}(v, 2-s, \chi) \\
a_{2 j, m}(v, 2-s, \chi) \\
\vdots \\
a_{h j, m}(v, 2-s, \chi)
\end{array}\right)
$$

für jedes $i$, und wegen (30), (34) ergibt sich

$$
(2 \pi|m|)^{s} \Gamma(s)^{-1}\left(\begin{array}{c}
\phi_{1 j}(s, \chi, m) \\
\phi_{2 j}(s, \chi, m) \\
\vdots \\
\phi_{h j}(s, \chi, m)
\end{array}\right)=\Phi(s, \chi)(2 \pi|m|)^{2-s} \Gamma(2-s)^{-1}\left(\begin{array}{c}
\phi_{1 j}(2-s, \chi, m) \\
\phi_{2 j}(2-s, \chi, m) \\
\vdots \\
\phi_{h j}(2-s, \chi, m)
\end{array}\right)
$$

Diese Formel kann als eine in vektorieller Form geschriebene Funktionalgleichung der Dirichletschen Reihen (31) verstanden werden.

\section{LITERATURVERZEICHNIS}

[1] A. Borel-G.D. Mostow: Alegbraic groups and discontinuous subgroups, Proc. Symp. Pure Math. IX, A.M.S. 1966.

[2] S. Helgason: Differential geometry and symmetric spaces, New York-London 1962.

[ 3 ] T. Kubota: On a special kind of Dirichlet series, to appear.

[4] R.P. Langlands: On the functional equations satisfied by Eisenstein series, to appear.

5) Solange keine Verwechselung möglich ist, verwenden wir den Buchstaben $\Gamma$ gleichzeitig für die Gammafunktion und für diskontinuierliche Gruppen. 
[5] E. Picard: Sur un groupe de transformation des points de l'espace situés du meme coté d'un plan, Bull. Soc. Math. de France 12 (1884), 43-47.

[6] A. Selberg: Harmonic analysis and discontinuous groups in weakly symmetric riemannian spaces with application to Dirichlet series, J. Indian Math. Soc. 20 (1956), 47-87.

[ 7 ] A. Selberg: Discontinuous groups and harmonic analysis, Proc. Int. Congr. 1962, 177-189.

Mathematisches Institut, Universitat zu Nagoya

und

Mathematisches Institut

der Technischen Hochschule Karlsruhe 